\title{
A culture model for neurite regeneration of human spinal cord neurons
}

\author{
T. Koechling a,b,c , H. Khalique ${ }^{a}$, E. Sundström ${ }^{d}$, J. Ávila ${ }^{b, c}$, F. Lima ${ }^{a} * *$ \\ a Departamento de Biología Molecular, Universidad Autónoma de Madrid, 28049 Madrid, Spain \\ b Centro de Biología Molecular Severo Ochoa (CSIC-UAM), 28049 Madrid, Spain \\ c CIBERNED, Centro de Investigación Biomédica en Red de Enfermedades Neurodegenerativas, Spain \\ d Neurotec Department, Division of Neurodegeneration and Neuroinflammation, Karolinska Institutet, Stockholm, Sweden
}

\section{A R T I C L E I N F O}

\section{Article history:}

Received 20 June 2011

Received in revised form 6 August 2011

Accepted 12 August 2011

\section{Keywords:}

Human neurite regeneration in culture

Human neural precursors

Postmitotic human neurons

Brain-derived neurotrophic factor

\begin{abstract}
A B S T R A C T
Effective therapeutic interventions for injuries of the central nervous system such as spinal cord injury are still unavailable, having a great impact on the quality of life of victims and their families, as well as high costs in medical care. Animal models of spinal cord injury are costly, time-consuming and laborintensive, making them unsuitable for screening large numbers of experimental conditions. Thus, culture models that recapitulate key aspects of neuronal changes in central nervous system injuries are needed to gain further understanding of the pathological and regenerative mechanisms involved, as well as to accelerate the screening of potential therapeutic agents. In this study we differentiated adherent cultures of dissociated human fetal spinal cord neural precursors into postmitotic neurons which we could then detach from culture plates and successfully freeze down in a viable state. When replated in neuronal medium without neurodifferentiating factors, these ready-to-use human spinal cord neurons remained viable, postmitotic and regenerated neurites in a cell density-dependent manner. Insulin-like growth factor 1 and growth hormone had no effect on neurite regeneration while brain-derived neurotrophic factor increased both the number of cells with neurites as well as the average neurite length. Our model can be applied to investigate factors involved in neuroregeneration of the human spinal cord and since adherent dissociated cell cultures are used, this system has significant potential as a screening platform for therapeutic agents to treat spinal cord injury.
\end{abstract}

(c) 2011 Elsevier B.V. All rights reserved.

\section{Introduction}

While injured peripheral nervous system neurons often readily regenerate, in central nervous system lesions such as spinal cord injury, this capacity is severely impaired by glial scar formation and inhibiting factors at the injury site (Yiu and He, 2006). However, it has been shown that removal of such inhibitory influences is insufficient to permit regeneration of central nervous system neurons (Steeves et al., 1998; Woolf, 2003) and intrinsic factors acting from

Abbreviations: DMEM, Dulbecco's modified Eagle's medium; bFGF, basic fibroblast growth factor; EGF, epidermal growth factor; IGF-1, insulin-like growth factor 1; BDNF, brain-derived neurotrophic factor; LIF, leukemia inhibitory factor; SAG, Smoothened agonist (SAG); DAPI, 4',6-diamidino-2-phenylindole (DAPI); GSK-3, glycogen synthase kinase 3; GAPDH, glyceraldehyde 3-phosphate dehydrogenase; hGH, human growth hormone (hGH); HRP, horseradish peroxidase (HRP); PBS, phosphate-buffered saline; SDS, sodium dodecyl sulphate (SDS).

* Corresponding author at: CV303, Department of Molecular Biology, Calle Francisco Tomás y Valiente 7, Universidad Autónoma de Madrid, 28049 Cantoblanco, Madrid, Spain. Tel.: +34 914977624; fax: +34 914973475.

E-mail address: filip.lim@uam.es (F. Lim). within the damaged neurons as well as insufficient availability of trophic factors also play an important role in regenerative failure.

Numerous animal models have been developed to study central nervous system neuroregeneration, particularly in spinal cord injury, but their high cost, labor-intensive execution and ethical issues preclude their use in high-throughput screening for therapeutic agents or as routine assays in experiments for elucidating molecular mechanisms. Furthermore, problems with extrapolating whole animal data to neuronal repair in human spinal cord injury (Akhtar et al., 2008) has highlighted the need to study the component mechanisms of neuroregeneration in simpler systems such as culture models. For example, dissociated retinal ganglion cells have been used extensively to study axonal regeneration (Lilley and Robbins, 2005) due to the ease of preparing axotomized neurons from rodents by optic nerve transection. However, the question remains of whether there are important species-specific differences in the regeneration of rodent central nervous system neurons compared to their human counterparts, and more importantly, how well retinal ganglion cells can model neuroregenerative mechanisms in the spinal cord. Human retinal ganglion cell cultures have been used to study axonal regeneration (Hopkins and Bunge, 1991; Takano et al., 2002; Tonges et al., 2011) but this is 
not feasible for high sample throughput due to the limited availability of human retinal biopsy or autopsy samples. Human spinal cord neurons derived from embryonic tissue have been cultured as dissociated monolayers, permitting characterization of various properties (Kato et al., 1985), but since neurons are postmitotic and cannot be propagated, routine use of such cultures would also depend on the constant availability of aborted tissue, with associated ethical problems. Another approach would be to culture neural precursor cells, which can then be differentiated in vitro to yield neurons. Indeed, recently human embryonic stem cells cultured as neurospheres were differentiated into sensory neurons which were then used as a model for neurite regeneration (Ziegler et al., 2010). In previous work, one of us (E. Sundström) has demonstrated that neural precursors derived from human fetal spinal cord can be maintained as neurosphere cultures over long periods without losing their capacity to differentiate into neurons (Åkesson et al., 2007). In the present study we describe culture methods to maintain human spinal cord neural precursors as dissociated cell monolayers and differentiate them into postmitotic neurons which can then be detached and stored frozen as "ready-to-use" neurons for studies of neuroregeneration at some later date. Cells in our model behaved similarly to other cultured central nervous system neurons, showing dependence of survival and neuritogenesis on cell density and neurotrophins such as brain-derived neurotrophic factor. Their simple controlled, homogeneous culture and quantitative neurodifferentiation offer great potential for this model to study neuroregenerative mechanisms and develop therapeutic approaches for human spinal cord injury.

\section{Materials and methods}

\subsection{Antibodies and reagents}

Dulbecco's modified Eagle's medium (DMEM)/F12, N-2 supplement, B27 supplement and TrypLE trypsin replacement were obtained from Gibco/Invitrogen (Barcelona, Spain). Recombinant human basic fibroblast growth factor (bFGF), epidermal growth factor (EGF), and insulin-like growth factor 1 (IGF-1) were from PeproTech (Rocky Hill, NJ). Human recombinant brain derived neurotrophic factor (BDNF), leukemia inhibitory factor (LIF) and forskolin were purchased from Alomone Labs (Jerusalem, Israel). Retinoic acid and the monoclonal anti-Neurofilament 160 antibody were from Sigma (Madrid, Spain). Smoothened agonist (SAG, catalog number 566660) and 4',6-Diamidino-2-phenylindole (DAPI) were obtained from Merck/Calbiochem (Darmstadt, Germany). The glycogen synthase kinase 3 (GSK-3) inhibitor SB216763 and Bax channel blocker were purchased from Tocris Cookson (Ellisville, MO). BD Matrigel Basement Membrane Matrix and the monoclonal Ki-67 antibody were from BD Biosciences/Pharmingen (Madrid, Spain). Monoclonal antibodies directed against nestin, Tau-1 and Tau-5 were from Millipore (Madrid, Spain) while those specific for PGP9.5, Bmi1 and glyceraldehyde 3-phosphate dehydrogenase (GAPDH, loading control) were obtained from Abcam (Cambridge, United Kingdom). The monoclonal SMI-31 antibody was from Covance/Sternberger Monoclonals (Baltimore, MD; (Sternberger and Sternberger, 1983). The 195 antiserum against $\beta$ III-tubulin and the 514 antibody, recognizing high molecular weight microtubuleassociated protein 2 (MAP2) isoforms were previously described (Sánchez Martin et al., 1998). Human growth hormone (hGH) was from Merck Serono (Geneva, Switzerland). Alexa Fluor 594 and Alexa Fluor 488 conjugated secondary antibodies used for immunostaining were obtained from Molecular probes (Eugene, OR) and horseradish peroxidase (HRP) conjugated secondary antibodies for Western blotting were from Santa Cruz Biotechnology (Santa Cruz, CA).

\subsection{Cell culture}

Human fetal neural precursor cells in the form of neurospheres were previously described (Åkesson et al., 2007). These cells were derived from human first trimester spinal cord tissue (Piao et al., 2006). In the present study we changed the original protocol from neurosphere culture in suspension to adherent growth on $60 \mathrm{~mm}$ culture dishes coated with basement membrane (Matrigel from BD Biosciences, Durham, NC) and on Matrigel-coated cover slips in 24 multi well plates. Free-floating neurospheres were digested in TrypLE for $5 \mathrm{~min}$ at $37^{\circ} \mathrm{C}$ and thereafter triturated gently into single cells using a cut-off $1 \mathrm{ml}$ plastic pipette tip, taking care not to introduce air during the procedure. Cells were propagated in stem cell medium, containing bFGF and EGF (composition of stem cell medium: DMEM/F12 medium with Glutamax (Gibco, Barcelona, Spain), supplemented with 0.5\% Albumax, $5 \mathrm{mM}$ HEPES, 0.6\% glucose, $1 \times \mathrm{N}-2,10 \mathrm{ng} / \mathrm{ml} \mathrm{bFGF}, 10 \mathrm{ng} / \mathrm{ml}$ EGF, $2 \mu \mathrm{g} / \mathrm{ml}$ heparin, non essential amino acids mixture and antibiotics $(20 \mathrm{mg} / \mathrm{ml}$ streptomycin, 20 units/ml penicillin). Cells were split after detachment with TrypLE, centrifugation and resuspension in stem cell medium. Cells were reseeded at concentrations superior to 200 cells $/ \mathrm{mm}^{2}$ and always maintained at relatively high densities after observing very restricted growth potential at low densities. Applying this routine, cultures were passaged once to twice a week and medium was changed every two days. Growth kinetics of the cultures were calculated by determining the number of population doublings over a defined period of time: the number of population doublings $=\log _{10}\left(n / n_{0}\right) \times\left[1 / \log _{10}(2)\right]$, with $n_{0}$ being the number of initially plated cells and $n$ being the number of cells at the end of the period of growth.

\subsection{Neuronal differentiation}

800,000 proliferative neural precursors were seeded per Matrigel-coated $60 \mathrm{~mm}$ culture dish (seeding density: 280 cells $/ \mathrm{mm}^{2}$ ) and incubated in supplemented neuronal medium (DMEM/F12 medium, supplemented with $1 \times \mathrm{B} 27,1 \times \mathrm{N}-2$, and streptomycin/penicillin) without bFGF and EGF for two weeks. For the first 7 days $5 \mu \mathrm{M}$ forskolin and $1 \mu \mathrm{M}$ retinoic acid were added to the medium, changing to a regimen of $0.1 \mu \mathrm{M}$ SAG and $1 \mu \mathrm{M}$ retinoic acid throughout the second week of treatment. Differentiation medium was changed every other day until completing the procedure after 14 days.

\subsection{Freezing of fetal human spinal cord neural precursors}

Cells were detached from a $60 \mathrm{~mm}$ culture dish with TrypLE and pelleted in a desktop centrifuge. Supernatant was discarded and the cell pellet was gently resuspended in $340 \mu \mathrm{l}$ stem cell medium. The resulting suspension was pipetted into a Nunc cryotube (Thermo Fisher Scientific, Roskilde, Denmark) containing $60 \mu \mathrm{l}$ dimethylsulfoxide, swirling the tube gently to homogenize the mixture. Cryotubes were then deposited in a Nalgene 5100 Cryo $1{ }^{\circ} \mathrm{C}$ freezing container (Thermo Fisher Scientific) and stored overnight in a $-80^{\circ} \mathrm{C}$ freezer, which froze the cells down gradually at a rate of $1{ }^{\circ} \mathrm{C} / \mathrm{min}$. The next day the frozen cultures were quickly transferred into liquid nitrogen containers and stored until further use. For recovery from frozen storage, cells were transferred on dry ice to the culture lab and thawed in a $37^{\circ} \mathrm{C}$ water bath before transfer to a centrifuge tube containing pre-warmed stem cell medium and removal of the freezing medium by centrifugation.

\subsection{Neurite regeneration experiments}

Postmitotic differentiated neurons derived from fetal human spinal cord neural precursors were detached from culture plates by 
mechanical and chemical means (scraping and incubation with TrypLE) and subsequently cryopreserved as described above, divided into aliquots of 200,000 cells for future use in regeneration experiments. Once thawed, cells were immediately separated from freezing medium and resuspended in neuronal medium without growth or differentiation inducing factors. The cell suspension was diluted and then replated on Matrigel-coated coverslips in 24 multi-well culture plates according to the desired cell densities of the regenerative cultures. After incubation for 5 days, the cultures were fixed and subjected to $\beta$ III-tubulin immunostaining for neurite identification and analysis by fluorescence microscopy.

\subsection{Western blot analysis}

Cells were washed with phosphate-buffered saline (PBS) and placed on ice. Using a plastic scraper, cells were homogenized in lysis buffer, containing $20 \mathrm{mM}$ HEPES, pH 7.4; $100 \mathrm{mM} \mathrm{NaCl}$; $100 \mathrm{mM}$ NaF; $1 \mathrm{mM} \mathrm{Na} \mathrm{VO}_{4} ; 5 \mathrm{mM}$ EDTA; $1 \%$ Triton X-100 and Complete protease inhibitor cocktail (Roche Diagnostics, Barcelona, Spain). After determining the protein content via the DC protein assay (Bio-Rad Laboratories SA, Madrid, Spain), samples were mixed with electrophoresis buffer containing sodium dodecyl sulphate (SDS), and boiled for $5 \mathrm{~min}$. $25 \mu \mathrm{g}$ of protein of each sample was separated on an 6-10\% polyacrylamide gel (depending on the molecular weight of the targeted protein) and subsequently blotted to a nitrocellulose membrane. Membranes were blocked with $10 \%$ skimmed milk in PBS containing 0.2\% Tween-20 (PBST) and subsequently incubated with the primary antibody diluted in PBST overnight at $4{ }^{\circ} \mathrm{C}$. After three washes with PBST the membranes were incubated with HRP-conjugated secondary anti-mouse or anti-rabbit antibody for $1 \mathrm{~h}$ at room temperature. Proteins reactive with these antibodies were visualized using an enhanced chemiluminescence detection system (PerkinElmer, Boston, MA) on a Gel Logic 1500 Imaging System (Eastman Kodak, Rochester, NY) and quantified with the ImageJ software (Abramoff et al., 2004).

\subsection{Immunocytochemistry}

For immunocytochemistry progenitor cells were cultured on cover slips in 24 well plates. Cell cultures were fixed with PBSbuffered $4 \%$ paraformaldehyde for $20 \mathrm{~min}$, followed by three washes with PBS. For the nuclear antigen Ki-67 a permeabilization step was introduced, consisting of $10 \mathrm{~min}$ incubation in $0.1 \%$ SDS in PBS. Subsequently the samples were blocked with blocking buffer (PBS, $1 \%$ fetal bovine serum, $0.2 \%$ Triton $\mathrm{X}-100$ ) for $1 \mathrm{~h}$ at room temperature. Cells were incubated with the primary antibody diluted in blocking buffer for $1 \mathrm{~h}$ at room temperature, followed by three washes with PBS for 5 min each. Primary antibodies used were: monoclonal anti-Neurofilament 160 antibody from Sigma (Madrid, Spain); monoclonal Ki-67 antibody from BD Biosciences/Pharmingen (Madrid, Spain); monoclonal antibodies directed against nestin, Tau-1 and Tau-5 from Millipore (Madrid, Spain); monoclonal antibodies specific for PGP9.5, Bmi1 and GAPDH obtained from Abcam (Cambridge, United Kingdom); monoclonal SMI-31 antibody from Covance/Sternberger Monoclonals (Baltimore, MD; the 195 antiserum against $\beta$ IIItubulin and the 514 antibody, recognizing high molecular weight microtubule-associated protein 2 (MAP2) isoforms previously described (Sánchez Martin et al., 1998). Samples were then incubated with the corresponding Alexa Fluor 594 or Alexa Fluor 488 conjugated secondary antibodies for $1 \mathrm{~h}$ at room temperature and then washed with PBS. Cell nuclei were counterstained with DAPI $(1 \mathrm{ng} / \mu \mathrm{l})$ for $10 \mathrm{~min}$ at room temperature and the samples were washed again. Coverslips were air-dried and then mounted on microscope slides with Fluoromount-G(SouthernBiotech, Birmingham, AL).

\subsection{Image and data analysis}

Samples were examined under a Zeiss Axioskop2 plus epifluorescence microscope. Images were recorded with a charge-coupled device Coolsnap FX color camera (Roper Scientific, Tucson, AZ) using Metavue 5.07 software (Molecular Devices, Downingtown, PA). Image analysis was carried out with ImageJ using the NeuronJ plug-in, which allows for neurite tracing and measurement as well as cell body area measurement (Meijering et al., 2004). Ten independent and randomly selected fields were analyzed and the following parameters were measured: number of cells (DAPI-positive nuclei), number of neurite-bearing cells, number of neurites per cell, length of neurites, cell body area.

\section{Results}

\subsection{Adherent monolayer culture of human fetal spinal cord neural precursor cells}

Human spinal cord neural precursors can be grown in long-term culture as neurospheres, conserving their neurodifferentiation potential for up to 18 passages (Åkesson et al., 2007). However, we experienced a number of difficulties with the neurosphere culture method associated with the aggregation of cells: speheres are difficult to dissociate without high mortality, complicating passaging, cell counting and ensuring homogeneous substrate availability to all cells in the sphere, or spheres of different sizes. To overcome these hurdles we modified the original neurosphere culture method for human fetal spinal cord neural precursors (Åkesson et al., 2007) to generate a culture model more easily set up and maintained (Fig. 1). Human fetal spinal cord neurospheres (Fig. 1A) were dissociated and seeded on culture plates covered with Matrigel basement membrane extracellular matrix to obtain adherent monolayers (Fig. 1C-F). This culture protocol permitted the homogeneous and quantitative expansion of neural precursors, facilitating the controlled differentiation towards neuronal cell types. Under proliferative culture conditions in stem cell medium (containing bFGF and EGF, see Section 2), cell populations doubled every five to six days (Fig. 1B) and leukemia inhibitory factor (LIF) was not necessary to maintain proliferative potential: the growth curves for cell cultures both with (filled blue circles) and without (open red triangles) LIF showed equal variances ( $F$-test: $F=1.12 ; p=0.43$ ) and a two sample $t$-test for equal variances confirmed the statistically significant coincidence of both growth curves $(p=0.98)$. Throughout this adherent culture procedure the cells expressed the proliferation marker Ki67 (Fig. 1E) and maintained their neurogenic capacity, as ascertained by their ability to differentiate into neurons (Fig. 1D and F) as described in more detail in the following section (see also Figs. 2 and 3).

\subsection{Differentiation of human spinal cord neurons}

Neurons were obtained from human fetal spinal cord neural precursors (Fig. 2) by changing the medium from stem cell medium to neuronal medium (see Section 2), thus withdrawing treatment with the mitotic factors bFGF and EGF while simultaneously commencing exposure to 3 differentiation inducing factors throughout 14 days: for the first 7 days of differentiation cells were incubated in neuronal medium in the presence of forskolin and retinoic acid, followed by exposure for a further 7 days to the combination of retinoic acid and SAG, a small molecule Smoothened agonist (Chen 

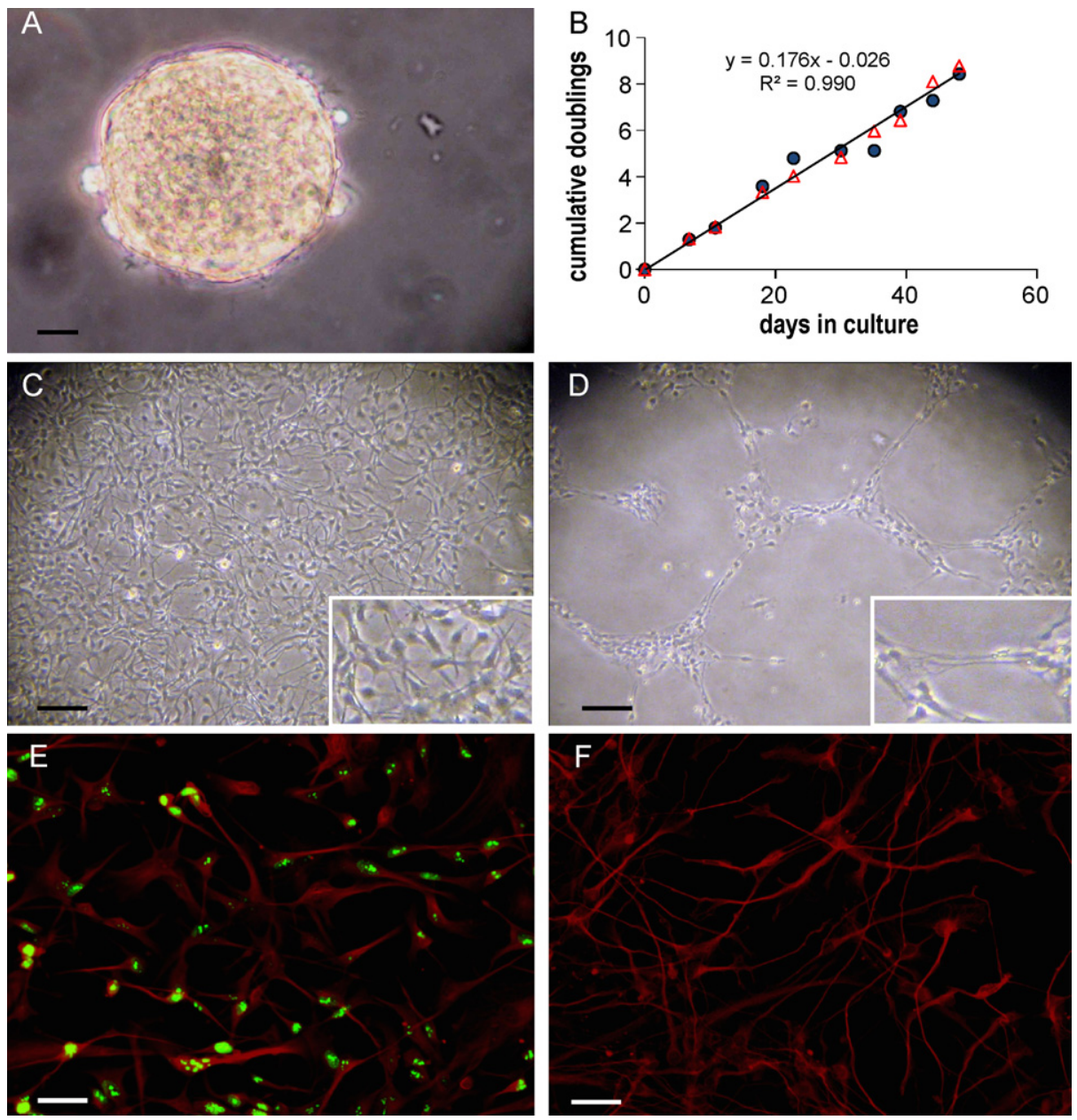

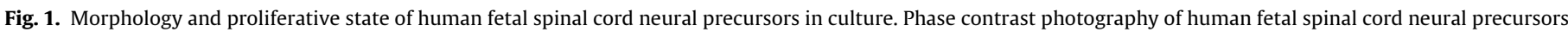

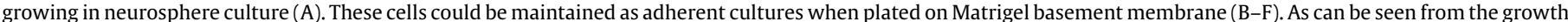

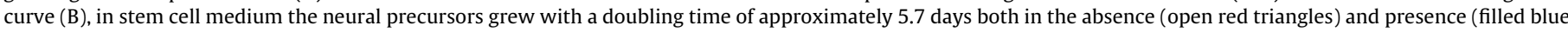

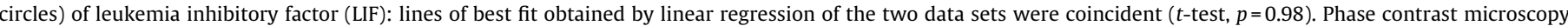

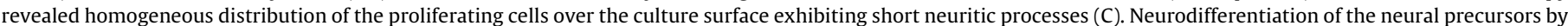

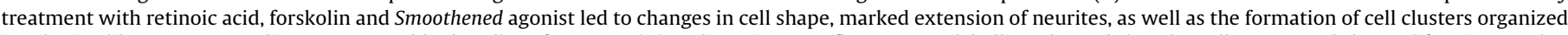

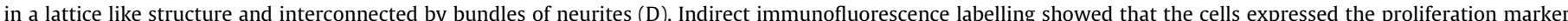

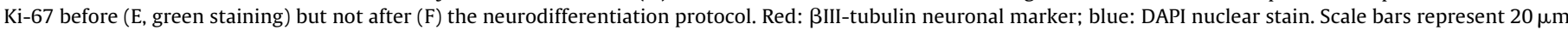
$(\mathrm{A}, \mathrm{E}$ and $\mathrm{F}), 100 \mu \mathrm{m}(\mathrm{C}, \mathrm{D})$. (For interpretation of the references to color in this figure legend, the reader is referred to the web version of the article.)

et al., 2002). Using both immunocytochemistry (Fig. 2) and Western blot analysis (Fig. 3) we confirmed neuronal differentiation of the spinal cord neural precursors by detection of upregulation of the neuronal marker $\beta$ III-tubulin and the simultaneous downregulation of the stem cell markers nestin and Bmi1 (Fig. 2A-D). By immunochemistry no consistent differences in staining intensity were detected using the neuron-specific antibodies PGP9.5 (data not shown), NF160 (Fig. 2E and F), Tau-1 (Fig. 2G and H) or Tau5 (data not shown). On the contrary, Western blots of extracts from differentiated cells compared to the untreated neural precursors showed an increase in the signal detected by each of these 4 antibodies (Fig. 3) which was significant for Tau-1 $(p<0.01)$, Tau-5 $(p<0.005)$ and $\beta$ III-tubulin $(p<0.05)$ but not significant for PGP9.5 or NF160. Cell morphology was also altered by the treatment: differentiated cells exhibited smaller cell bodies and longer neuritic processes (see next section and Fig. 4) as well as the formation of grid-like structures interconnecting different cell clusters (Fig. 1D). After differentiation, neurons were completely negative in immunostaining for the proliferation marker Ki-67 (Fig. 1F), confirming their postmitotic state, while the hNPCs under proliferative culture conditions were clearly Ki-67-immunopositive (Fig. 1E).

\subsection{Regeneration of postmitotic human spinal cord neurons is dependent on differentiation state and replating density}

We next evaluated if the postmitotic, differentiated hNPCs were able to survive dissociation and detachment from culture plates and storage in liquid nitrogen. Using our optimized freezing protocol (see Section 2) we have been able to store these in vitrodifferentiated human spinal cord neurons in liquid nitrogen for at least 12 months with a viability of $\sim 70 \%$. When thawed, aliquots of these pre-differentiated neural precursors were replated onto Matrigel-coated plates in neuronal medium (Fig. 4A), where their neuronal morphology was regenerated even in the absence of the neurodifferentiating factors retinoic acid, forskolin and SAG (Fig. 4B and $C$ ). For each number of cells plated, we quantified the number of cells present after 5 days (Fig. 4D), those re-extending neurites (Fig. 4E), the average length of neurites (Fig. 4F) and the cell body area (Fig. 4G), observing clear differences for these parameters between proliferating precursor cells (PRO, open circles) and differentiated neurons (DIF, filled circles). The percentage of the differentiated and replated cells that re-grew neurites was almost twice that of the proliferative cells (Fig. 4E), with the length of these neuritic processes being $30 \%$ longer in case of the regenerating 

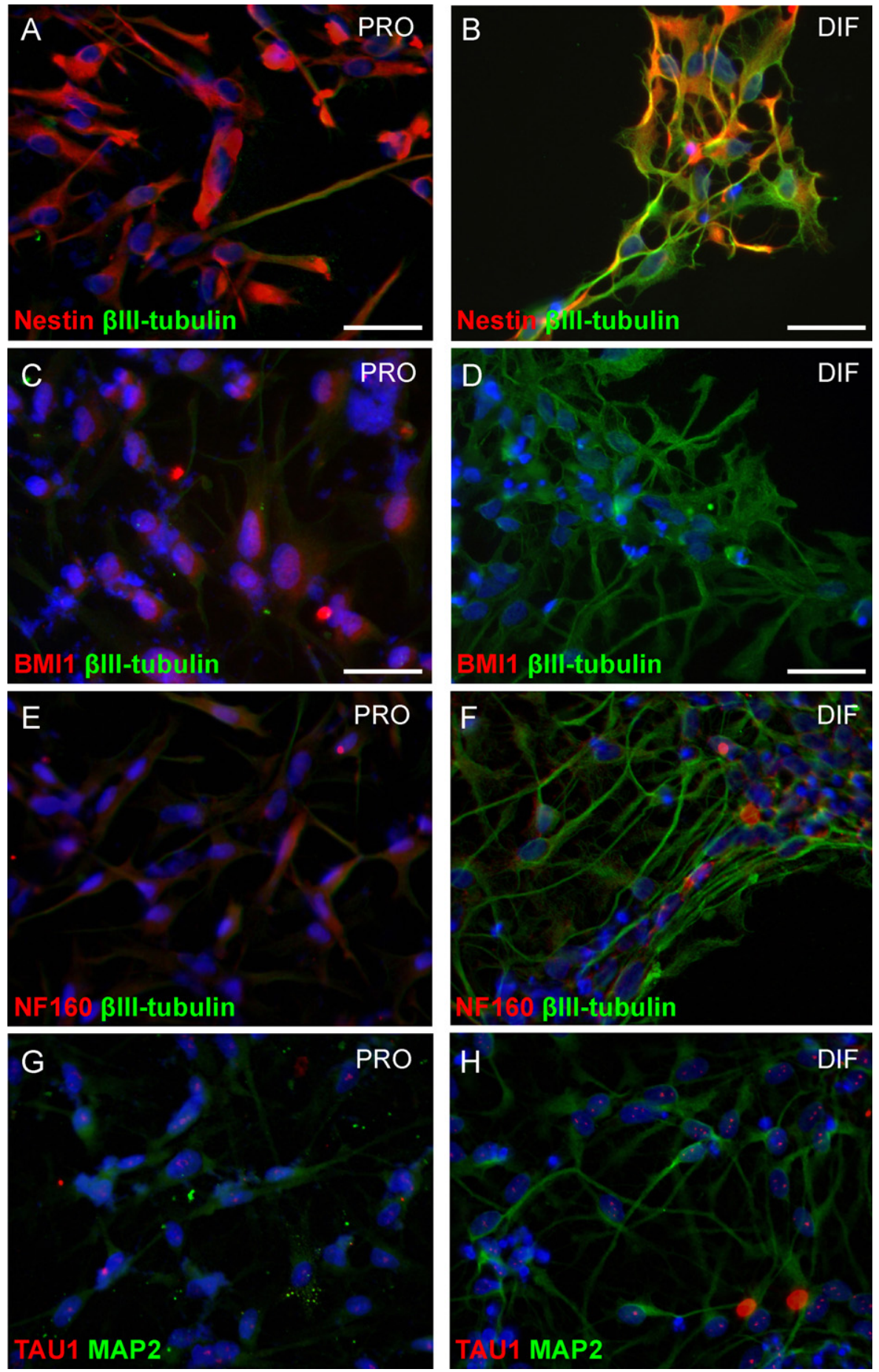

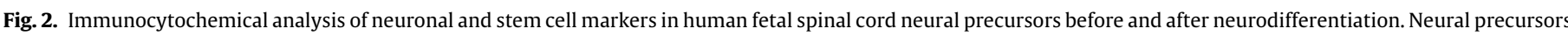

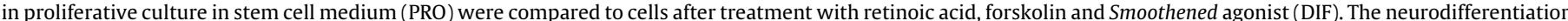

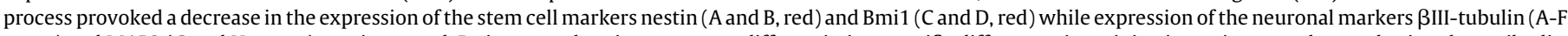

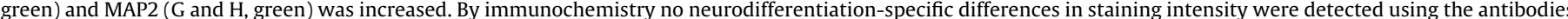

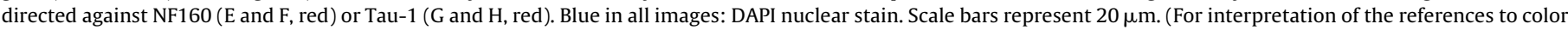
in this figure legend, the reader is referred to the web version of the article.)

cells (Fig. 4F). Furthermore, the bodies of the proliferative neural precursors were significantly larger than those of the neuronally differentiated and regenerating cells $(589 \mu \mathrm{m} \pm 58 \mu \mathrm{m}$ compared to $256 \mu \mathrm{m} \pm 22 \mu \mathrm{m}, p<0.005$, Fig. $4 \mathrm{G}$ ).

We next examined in more detail the differentiated human spinal cord neurons after replating. Our results show that the ability of these cells to re-extend neurites is affected by the reseeding cell density (Fig. 4B, C and E). The low density cultures resembled clonal culture conditions in which no cell-cell contact occurred (Fig. 4B), while at higher densities the distance between individual cells diminished and intercellular contact via neuritic outgrowths was observed (Fig. 4C). When cells were seeded at a range of different densities from 10 to 100 cells $/ \mathrm{mm}^{2}$, we observed up to $50 \%$ increase in the number of cells that extended neurites (Fig. 4E). The average length of the neurites (Fig. 4F) and the number of neurites per neurite-bearing cell (not shown), however, were not affected by seeding density. 
A
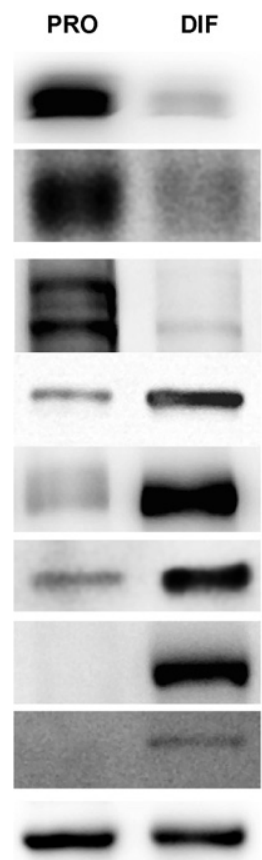

$52-68 \mathrm{~K}$

$52-68 \mathrm{~K}$

$36 \mathrm{~K}$

B

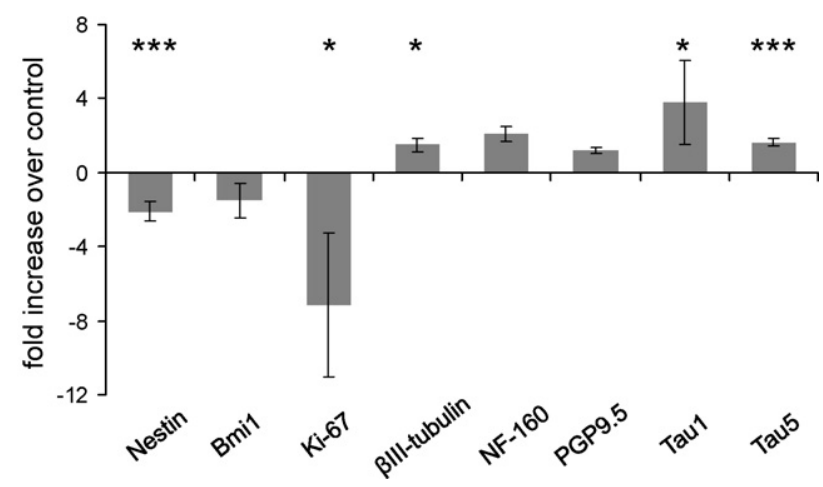

Fig. 3. Western blot analysis of neurodifferentiation of human fetal spinal cord neural precursors. Representative Western blots (A) showing high expression of the stem cell-specific antigens nestin and Bmi1, in proliferative human fetal spinal cord neural precursors (PRO) and which decreases after neuronal differentiation (DIF) while expression of the neuronal markers $\beta$ III-tubulin, NF-160, PGP9.5, Tau1 and Tau 5 is low in proliferative precursors and increases after differentiation. The proliferation marker Ki-67 was expressed in the precursor cells but not after differentiation into postmitotic neurons. Glyceraldehyde 3-phosphate dehydrogenase (GAPDH) was used as a loading control in each experiment. Quantification of the expression levels of the various markers was carried out by pixel analysis of the digital images (B). All values were normalized with respect to GAPDH levels in the same extracts. Values plotted in the histogram represent the fold change in expression level of each marker in the neurodifferentiated cells with respect to that in the precursors. Error bars represent the standard deviation of the mean of 3-5 independent experiments. ${ }^{*} p<0.05 ;{ }^{* * *} p<0.005$; ANOVA.

\subsection{Failure of neurite regeneration in low density spinal cord neuron cultures can be rescued by BDNF}

While nearly all of the human spinal cord neural cells bear neurites after our in vitro differentiation procedure, a large fraction of the cells after detachment and replating failed to re-extend neurites, indicating that this model recapitulates some of the changes resulting in dysfunctional neurons after lesions such as spinal cord injury. We postulated that neuroprotective agents might thus decrease the proportion of cells unable to regenerate neurites. To ensure a large fraction of non-regenerating cells we reseeded the differentiated postmitotic neurons at a low density $\left(25\right.$ cells $/ \mathrm{mm}^{2}$ ) and tested the effect of several neuroprotective agents known to act in different ways: SB216763 (a GSK3 inhibitor), 3,6-Dibromo$\alpha$-(1-piperazinylmethyl)-9H-carbazole-9-ethanol dihydrochloride (a Bax channel blocker), human growth hormone (hGH), insulinlike growth factor 1 (IGF-1) and brain-derived neurotrophic factor (BDNF). We observed no significant changes in neurite regeneration with SB216763 (2.5-7.5 $\mu \mathrm{M})$, Bax blocker (5-15 $\mu \mathrm{M})$, hGH (1-100 ng/ml) or IGF-1 (20-500 ng/ml) but BDNF (30 ng/ml) clearly enhanced neuroregeneration in this model (Fig. 5B), as shown immunocytochemically, provoking a $30 \%$ increase in cell survival (Fig. 5C), a 58\% increase in the number of cells that re-extended neurites (Fig. 5D) and 30\% increase in the average length of the neurites (Fig. 5E).

\section{Discussion}

\subsection{A simple and robust culture system for human spinal cord neuronal precursors}

Initial studies of human fetal spinal cord neural precursors cultured as neurospheres reported the loss of neurogenic differentiation capacity with successive passages (Quinn et al., 1999) but later work described long term culture conditions which conserve the potential of these cells to differentiate towards a neuronal fate for up to 18 passages (Åkesson et al., 2007). The neurosphere culture method, however, bears several inherent difficulties: high mortality upon sphere dissociation when passaging (over 90\% cell death) (Reynolds and Rietze, 2005); poor diffusion of nutrients into the core of the sphere resulting in nonhomogeneous substrate availability; cell aggregates make difficult the precise quantification of cell number of the sphere or sphere fragments. Furthermore the cell population of a neurosphere consists of different cell types, not all of which are neural precursors capable of differentiation into neurons (Jensen and Parmar, 2006). By successfully establishing an adherent dissociated cell culture system for human fetal spinal cord neural precursors and differentiated cells derived from them, we were able to identify individual neurons, their nuclei, soma and neurites, which permitted quantitative analysis of the neurons and their neurites by semi-automated image processing using Image (Abramoff et al., 2004) and NeuronJ (Meijering et al., 2004) software. Additionally, our dissociated adherent cultures permit the isolation of single cells for subsequent cloning, enabling the selection of single neural precursors with specific characteristics or after genetic manipulation. Leukemia Inhibitory Factor (LIF) was not necessary to maintain proliferation or the neurogenic capacity of our human spinal cord neural precursors; cell cultures grown without LIF showed the same proliferation kinetics as those grown in the presence of LIF. This is consistent with the observation that LIF has no additive or synergistic effect over bFGF on the proliferation of neural precursors derived from human embryonic stem cells (Zhang et al., 2001; Zhang and Zhang, 2010), and a recent report that LIF is not required for the growth of human fetal forebrain neural precursors (Sun et al., 2008). Thus another advantage of our system is that it avoids the need for labor-intensive feeder cell co-culture or expensive supplementation with purified LIF.

\subsection{A culture assay for neuroregeneration of postmitotic human spinal cord neurons}

Successful differentiation of the human spinal cord neural precursors in the neuronal lineage was confirmed by augmented expression of several neuron-specific epitopes, namely $\beta$ IIItubulin, Tau-1, Tau-5, Neurofilament-M and PGP9.5 and concurrent decreased expression of the markers nestin and Bmi1, which are enriched in stem cells, after two weeks of treatment with forskolin, 
A
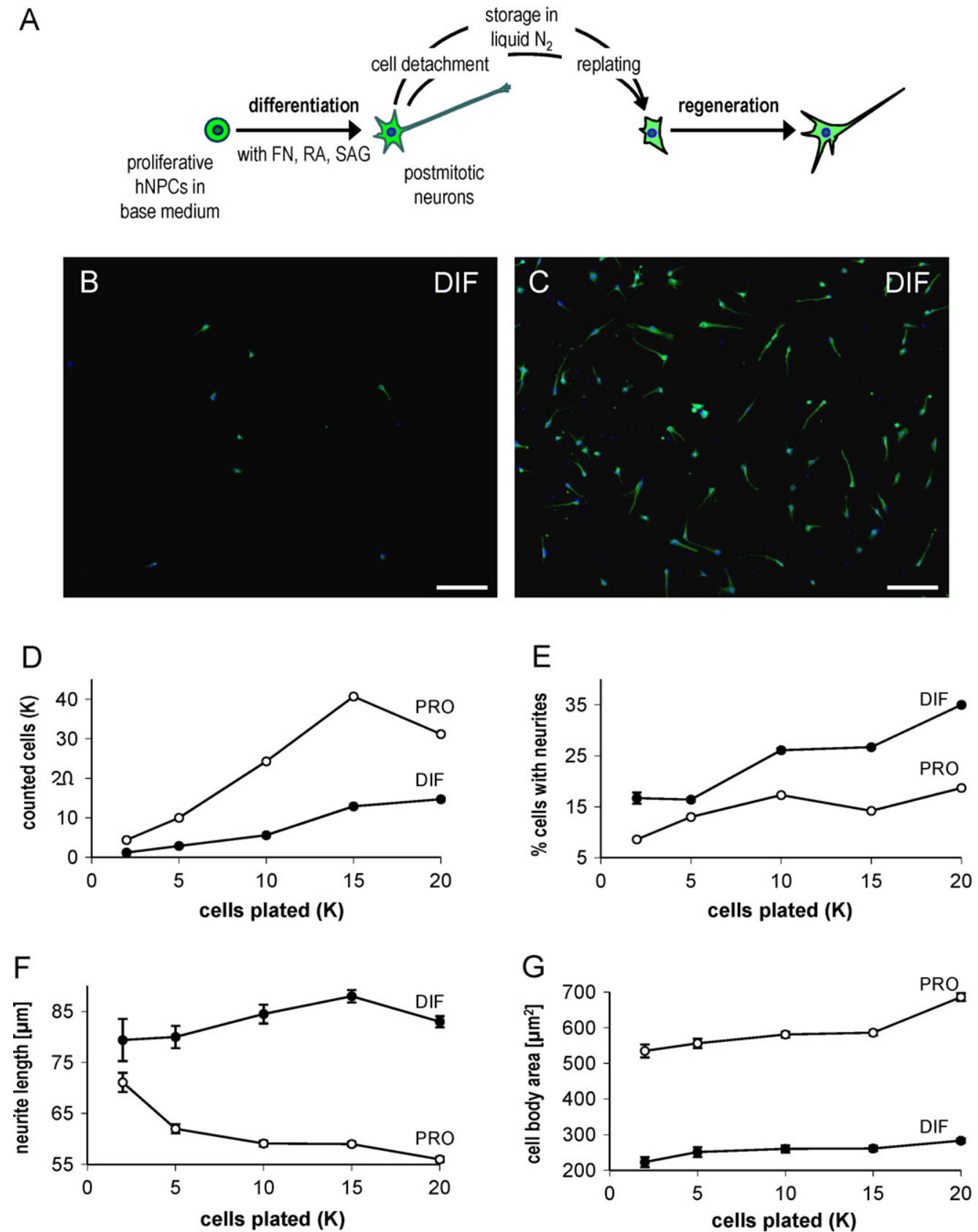

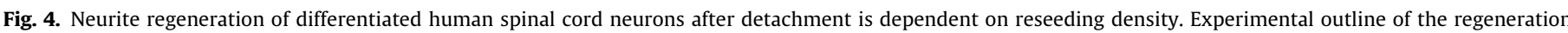

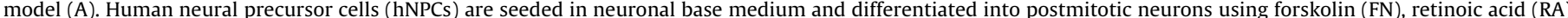

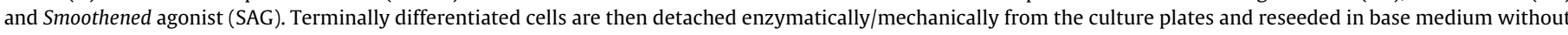

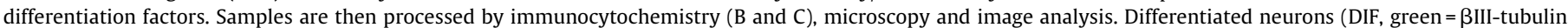

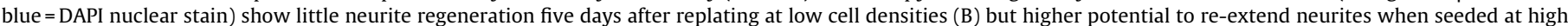

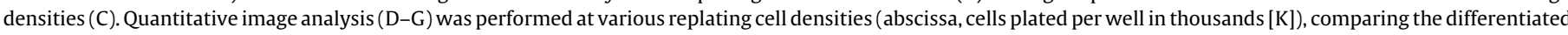

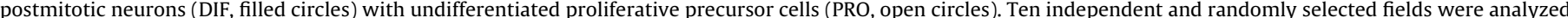

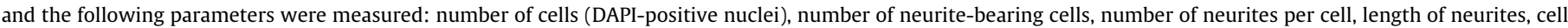

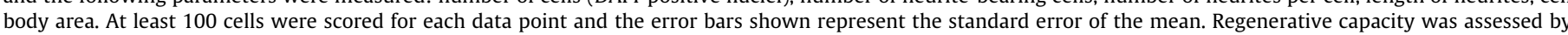

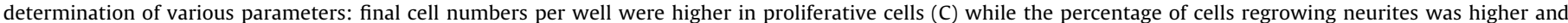

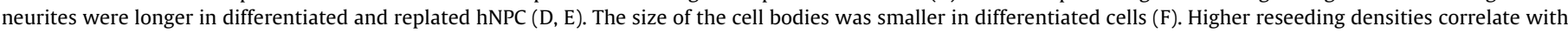

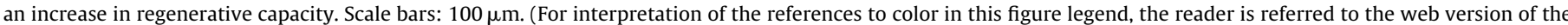
article.)

retinoic acid and Smoothened agonist. The postmitotic state of the neurons was confirmed by the absence of Ki-67 expression and furthermore, when replated in stem cell medium after having gone through the entire differentiation process, the cells did not revert to a proliferative state under these pro-mitotic conditions and remained negative for Ki67 staining (data not shown).
We next optimized procedures for detachment of these in vitrodifferentiated human spinal cord neurons from culture plates and their storage in frozen form to permit subsequent studies of regeneration of the neuronal morphology upon thawing and replating. Quantitative image analysis showed that the reseeded cells were morphologically different from the proliferative neural precursors, 

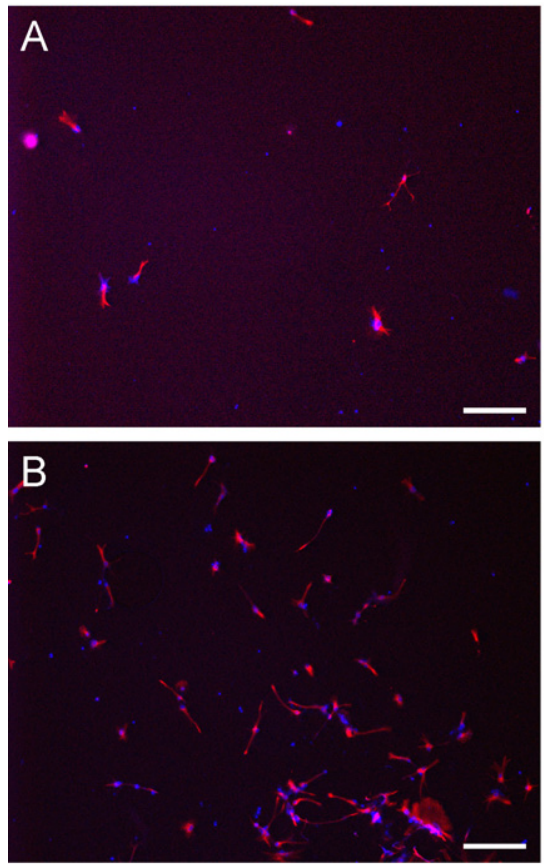

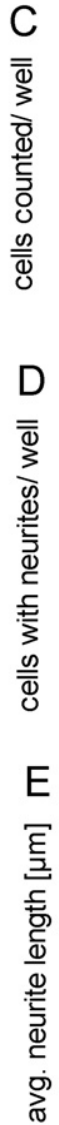

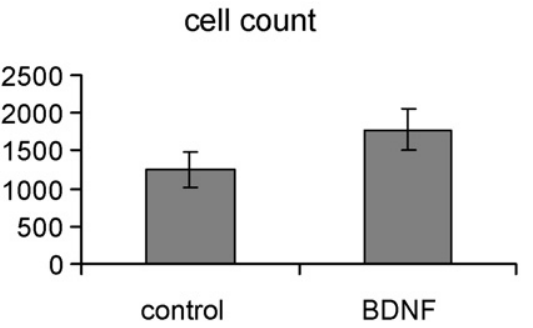

cells with neurites

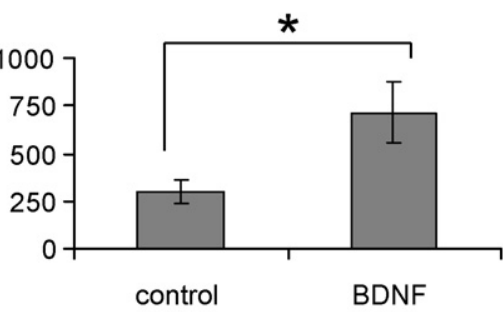

neurite length

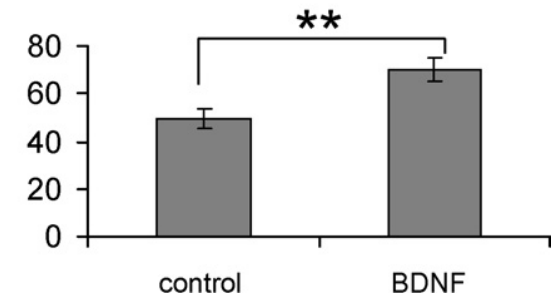

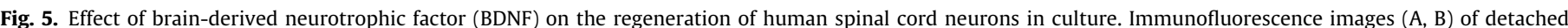

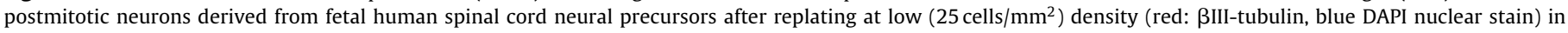

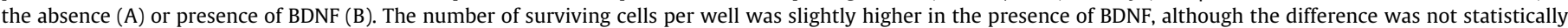

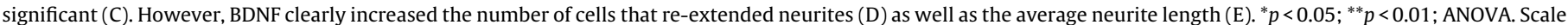
bars represent $100 \mu \mathrm{m}$. (For interpretation of the references to color in this figure legend, the reader is referred to the web version of the article.)

exhibiting smaller cell bodies, more cells with neurites, and longer neurites on average: together with our immunochemical data these results reveal the successful neurodifferentiation of human spinal cord neural precursors into postmitotic neurons.

\subsection{Identification of therapeutic candidates for human spinal cord repair}

Isolated neurons in our culture system derived from precursors originally located in the human spinal cord, have the potential for recapitulating many important aspects of human spinal cord injury but since other cell types such as glia are absent, it should be kept in mind that many of the inhibitory, or extrinsic factors are not modelled. On the other hand, this simplifies the study of the intrinsic factors playing a role in neuroregeneration as well as responses to trophic agents. Several intracellular components have been identified and much work is underway on how to manipulate them to favor a regenerative response of central nervous system axons in vivo: for example Bcl-2 (Jiao et al., 2005), mTOR (Park et al., 2008) and GSK-3 (Dill et al., 2008). Of the large number of soluble factors with trophic effects identified, so far only a few neurotrophins such as NGF, NT3 and BDNF have been investigated for therapeutic potential of damaged spinal cord neurons (Schwab, 2002), although other growth factors such as IGF-1 and growth hormone are also being examined for their therapeutic effects in the central nervous system (Devesa et al., 2009, 2010; Homma et al., 2007; Leinninger and Feldman, 2005; Smith et al., 1993). In our culture system inhibition of GSK-3 or Bax, or addition of IGF-1 or growth hormone did not significantly affect neurite regeneration, indicating that any possible therapeutic effects of these agents is not direct.

\subsection{BDNF can act directly on human spinal cord neurons to promote regeneration}

Detailed analysis of the pre-differentiated human spinal cord neurons showed that higher replating cell densities increased the number of neurite-bearing cells but not the average neurite length. Since this effect occurred at cell densities where many cells were still not in contact with neighbouring cells, it is likely to be due to autocrine or paracrine factors which improve neurite initiation but which do not affect neurite extension thereafter. Increased neurite regeneration at higher cell densities has also been observed in other culture assay systems such as the neuronal cell line NG108-15 (Mitchell et al., 2007) and in dissociated rat retinal ganglion neurons (Goldberg et al., 2002). However, as in the former example, this effect is often not quantified, or based on only one parameter, such as the length of the longest axon measured in the latter case (Goldberg et al., 2002). Significantly, in our system we found that although the neurotrophin BDNF also augmented neuroregeneration, its mode of action was different to the factor(s) responsible for the cell density-dependent effect. BDNF increased both neuritebearing cell number and the average neurite length, showing that this neurotrophin plays a role both in the initiation as well as the extension of neurites. The dissociated human spinal cord neurons in our system thus do not seem to secrete effective levels of BDNF, supporting the idea that another major factor in the fail- 
ure of central nervous system regeneration is the lack of trophic support (Widenfalk et al., 2001). BDNF is of special interest in central nervous system neuronal repair because accumulating evidence in rat models indicates that it may play a significant role in the phenomenon of axonal regrowth from dorsal root ganglia through spinal cord injury sites when the neurons are primed by a preconditioning lesion (Neumann and Woolf, 1999). BDNF is upregulated in dorsal root ganglia in response to spinal cord injury (Sebert and Shooter, 1993) and delivered by anterograde transport into the spinal cord (Tonra et al., 1998; Zhou and Rush, 1996) where it may be transferred transynaptically from neuron to neuron (von Bartheld, 2004). Furthermore, a recent report showed that BDNF antiserum blocked preconditioning-lesion induced central nervous system axonal regrowth while administration of BDNF to the peripheral nerve fibers of DRG neurons increased the regeneration of these neurons in the spinal cord (Song et al., 2008). Since other studies have shown that BDNF delivery directly into the spinal cord does not promote axonal recovery (Bradbury et al., 1998), our present results that isolated human spinal cord neurons in culture are indeed responsive to BDNF are important in supporting the idea that the nonresponsiveness to this neurotrophin in vivo is probably due to inhibitory effects in the spinal cord such as neutralization by binding to truncated trkB receptors on astrocytes (Fryer et al., 1997; Liebl et al., 2001).

Together with the capability of our system to generate largescale preparations of postmitotic human spinal cord neurons in a "readyto-use" state and the above results confirming that BDNF is of therapeutic interest for human spinal cord neurons, our present study highlights the potential of this culture model for neuroregeneration experiments and drug screening.

\section{Conflict of interest statement}

There are no conflicts of interest.

\section{Acknowledgments}

We are grateful to Carolina Vincelli and the Raul Gonzalez Salas Foundation for a research grant supporting this work.

\section{References}

Abramoff MD, Magelhaes PJ, Ram SJ. Image processing with image. J Biophot Int 2004; $11: 36-42$.

Åkesson E, Piao JH, Samuelsson EB, Holmberg L, Kjaeldgaard A, Falci S, Sundström E, Seiger A. Long-term culture and neuronal survival after intraspinal transplantation of human spinal cord-derived neurospheres. Physiol Behav 2007;92:60-6.

Akhtar AZ, Pippin JJ, Sandusky CB. Animal models in spinal cord injury: a review. Rev Neurosci 2008;19:47-60.

Bradbury EJ, King VR, Simmons LJ, Priestley JV, McMahon SB. NT-3, but not BDNF, prevents atrophy and death of axotomized spinal cord projection neurons. Eur J Neurosci 1998;10:3058-68.

Chen JK, Taipale J, Young KE, Maiti T, Beachy PA. Small molecule modulation of smoothened activity. Proc Natl Acad Sci USA 2002;99:14071-6.

Devesa J, Casteleiro N, Rodicio C, Lopez N, Reimunde P. Growth hormone deficiency and cerebral palsy. Ther Clin Risk Manag 2010;6:413-8.

Devesa J, Reimunde P, Devesa A, Souto S, Lopez-Amado M, Devesa P, Arce VM. Recovery from neurological sequelae secondary to oncological brain surgery in an adult growth hormone-deficient patient after growth hormone treatment. J Rehabil Med 2009;41:775-7.

Dill J, Wang H, Zhou F, Li S. Inactivation of glycogen synthase kinase 3 promotes axonal growth and recovery in the CNS. J Neurosci 2008;28:8914-28.

Fryer RH, Kaplan DR, Kromer LF. Truncated trkB receptors on nonneuronal cells inhibit BDNF-induced neurite outgrowth in vitro. Exp Neurol 1997;148:616-27.

Goldberg JL, Espinosa JS, Xu Y, Davidson N, Kovacs GT, Barres BA. Retinal ganglion cells do not extend axons by default: promotion by neurotrophic signaling and electrical activity. Neuron 2002;33:689-702.

Homma K, Koriyama Y, Mawatari K, Higuchi Y, Kosaka J, Kato S. Early downregulation of IGF-I decides the fate of rat retinal ganglion cells after optic nerve injury. Neurochem Int 2007;50:741-8.

Hopkins JM, Bunge RP. Regeneration of axons from adult human retina in vitro. Exp Neurol 1991;112:243-51.
Jensen JB, Parmar M. Strengths and limitations of the neurosphere culture system. Mol Neurobiol 2006;34:153-61.

Jiao J, Huang X, Feit-Leithman RA, Neve RL, Snider W, Dartt DA, Chen DF. Bcl-2 enhances $\mathrm{Ca}(2+)$ signaling to support the intrinsic regenerative capacity of CNS axons. EMBO J 2005;24:1068-78.

Kato AC, Touzeau G, Bertrand D, Bader CR. Human spinal cord neurons in dissociated monolayer cultures: morphological, biochemical, and electrophysiological properties. J Neurosci 1985;5:2750-61.

Leinninger GM, Feldman EL. Insulin-like growth factors in the treatment of neurological disease. Endocr Dev 2005;9:135-59.

Liebl DJ, Huang W, Young W, Parada LF. Regulation of Trk receptors following contusion of the rat spinal cord. Exp Neurol 2001;167:15-26.

Lilley S, Robbins J. The rat retinal ganglion cell in culture: an accessible CNS neurone. J Pharmacol Toxicol Methods 2005;51:209-20.

Meijering E, Jacob M, Sarria J-CF, Steiner P, Hirling H, Unser M. Design and validation of a tool for neurite tracing and analysis in fluorescence microscopy images. Cytometry A 2004;58:167-76.

Mitchell PJ, Hanson JC, Quets-Nguyen AT, Bergeron M, Smith RC. A quantitative method for analysis of in vitro neurite outgrowth. J Neurosci Methods 2007;164:350-62.

Neumann S, Woolf CJ. Regeneration of dorsal column fibers into and beyond the lesion site following adult spinal cord injury. Neuron 1999;23:83-91.

Park KK, Liu K, Hu Y, Smith PD, Wang C, Cai B, Xu B, Connolly L, Kramvis I, Sahin $\mathrm{M}$, He Z. Promoting axon regeneration in the adult CNS by modulation of the PTEN/mTOR pathway. Science 2008;322:963-6.

Piao JH, Odeberg J, Samuelsson EB, Kjaeldgaard A, Falci S, Seiger A, Sundström E, Åkesson E. Cellular composition of long-term human spinal cord- and forebrainderived neurosphere cultures. J Neurosci Res 2006;84:471-82.

Quinn SM, Walters WM, Vescovi AL, Whittemore SR. Lineage restriction of neuroepithelial precursor cells from fetal human spinal cord. J Neurosci Res 1999;57:590-602.

Reynolds BA, Rietze RL. Neural stem cells and neurospheres-re-evaluating the relationship. Nat Methods 2005;2:333-6.

Sánchez Martin C, Díaz-Nido J, Avila J. Regulation of a site-specific phosphorylation of the microtubule-associated protein 2 during the development of cultured neurons. Neuroscience 1998;87:861-70.

Schwab ME. Repairing the injured spinal cord. Science (New York, NY) 2002;295:1029-31.

Sebert ME, Shooter EM. Expression of mRNA for neurotrophic factors and their receptors in the rat dorsal root ganglion and sciatic nerve following nerve injury. J Neurosci Res 1993;36:357-67.

Smith RA, Melmed S, Sherman B, Frane J, Munsat TL, Festoff BW. Recombinant growth hormone treatment of amyotrophic lateral sclerosis. Muscle Nerve 1993;16:624-33.

Song XY, Li F, Zhang FH, Zhong JH, Zhou XF. Peripherally-derived BDNF promotes regeneration of ascending sensory neurons after spinal cord injury. PloS One 2008;3:e1707.

Steeves JD, Tetzlaff W. Engines, accelerators, and brakes on functional spinal cord repair. Ann NY Acad Sci 1998;860:412-24.

Sternberger LA, Sternberger NH. Monoclonal antibodies distinguish phosphorylated and nonphosphorylated forms of neurofilaments in situ. Proc Natl Acad Sci USA 1983;80:6126-30.

Sun Y, Pollard S, Conti L, Toselli M, Biella G, Parkin G, Willatt L, Falk A, Cattaneo E, Smith A. Long-term tripotent differentiation capacity of human neural stem (NS) cells in adherent culture. Mol Cell Neurosci 2008;38: 245-58.

Takano M, Horie H, Iijima Y, Dezawa M, Sawada H, Ishikawa Y. Brain-derived neurotrophic factor enhances neurite regeneration from retinal ganglion cells in aged human retina in vitro. Exp Eye Res 2002;74:319-23.

Tonges L, Ostendorf T, Lamballe F, Genestine M, Dono R, Koch JC, Bahr M, Maina F, Lingor P. Hepatocyte growth factor protects retinal ganglion cells by increasing neuronal survival and axonal regeneration in vitro and in vivo. J Neurochem 2011;117:892-903.

Tonra JR, Curtis R, Wong V, Cliffer KD, Park JS, Timmes A, Nguyen T, Lindsay RM, Acheson A, DiStefano PS. Axotomy upregulates the anterograde transport and expression of brain-derived neurotrophic factor by sensory neurons. J Neurosci 1998;18:4374-83.

von Bartheld CS. Axonal transport and neuronal transcytosis of trophic factors, tracers, and pathogens. J Neurobiol 2004;58:295-314.

Widenfalk J, Lundstromer K, Jubran M, Brene S, Olson L. Neurotrophic factors and receptors in the immature and adult spinal cord after mechanical injury or kainic acid. J Neurosci 2001;21:3457-75.

Woolf CJ. No Nogo: now where to go? Neuron 2003;38:153-6.

Yiu G, He Z. Glial inhibition of CNS axon regeneration. Nat Rev Neurosci 2006;7:617-27.

Zhang SC, Wernig M, Duncan ID, Brustle O, Thomson JA. In vitro differentiation of transplantable neural precursors from human embryonic stem cells. Nat Biotechnol 2001;19:1129-33.

Zhang XQ Zhang SC. Differentiation of neural precursors and dopaminergic neurons from human embryonic stem cells. Methods Mol Biol 2010;584:355-66.

Zhou XF, Rush RA. Endogenous brain-derived neurotrophic factor is anterogradely transported in primary sensory neurons. Neuroscience 1996;74: 945-53.

Ziegler L, Segal-Ruder Y, Coppola G, Reis A, Geschwind D, Fainzilber M, Goldstein RS. A human neuron injury model for molecular studies of axonal regeneration. Exp Neurol 2010;223:119-27. 